PSICOLOGÍA

IBEROAMERICANA
Psicología Iberoamericana ISSN: 1405-0943

revista.psicologia@ibero.mx

Universidad Iberoamericana, Ciudad de México México

\title{
Efectividad de una intervención de atención plena en enfermas crónicas con síntomas ansiosos y depresivos
}

Bernal Pérez, Rosalía Pilar; Berenzon Gorn, Shoshana; Tiburcio Sainz, Marcela

Efectividad de una intervención de atención plena en enfermas crónicas con síntomas ansiosos y depresivos

Psicología Iberoamericana, vol. 26, núm. 2, 2018

Universidad Iberoamericana, Ciudad de México, México

Disponible en: http://www.redalyc.org/articulo.oa?id=133959841006 


\title{
Efectividad de una intervención de atención plena en enfermas crónicas con síntomas ansiosos y depresivos
}

\author{
EFFECTIVENESS OF A MINDFULNESS INTERVENTION IN CHRONICALLY ILL FEMALE PATIENTS \\ WITH ANXIETY AND DEPRESSION SYMPTOMS
}

Rosalía Pilar Bernal Pérez

Universidad Nacional Autónoma de México, México

Redalyc: http://www.redalyc.org/articulo.oa?

pilar18_24@hotmail.com

Shoshana Berenzon Gorn

Instituto Nacional de Psiquiatría Ramón de la Fuente

Muñiz, México

Marcela Tiburcio Sainz

Instituto Nacional de Psiquiatría Ramón de la Fuente

Muñiz, México id $=133959841006$

Recepción: 09 Agosto 2018

Aprobación: 05 Marzo 2019

\section{RESUMEN:}

Se evaluó la efectividad de una intervención basada en la atención plena en mujeres con enfermedades crónicas, sintomatología ansiosa y depresiva en el primer nivel de atención. El objetivo de este tipo de intervenciones es desarrollar una atención deliberada a lo que se experimenta en el cuerpo, la mente y las emociones en el momento en que surgen, a manera de fomentar el bienestar. Se midieron 50 mujeres provenientes de dos centros de salud a través de los instrumentos: pss, hads y maas antes y después de la intervención. Los resultados mostraron diferencias estadísticamente significativas en la disminución de los niveles de estrés, sintomatología ansiosa, sintomatología depresiva y un aumento en la atención plena. Se obtuvo una intervención efectiva para el escenario y población propuesta.

PALABRAS CLAVE: Atención plena, estrés, síntomas ansiosos, síntomas depresivos, enfermas crónicas.

\section{Abstract:}

Thispaper assesses the effectiveness of a mindfulness-based intervention in women with chronic diseases, anxiety and depression symptoms in primary health care. The intervention focuses on what is experienced in the body, mind and emotions in the present moment, with an aim of improving overall well-being. Fifty participants from two health centers were evaluated before and after the intervention, using the following instruments: pss, hads and maas. The results show statistically significant differences in the reduction of stress levels, anxiety symptoms, depression symptoms and an increase in mindfulness. An effective intervention was obtained for the proposed setting and population.

KEYWORDS: Mindfulness, stress, anxiety and depressive symptoms, chronically ill.

\section{INTRODUCCIÓN}

Las enfermedades crónicas tales como los padecimientos cardíacos, las enfermedades respiratorias y la diabetes son las principales causas de mortalidad en el mundo, responsables del $63 \%$ de las muertes anuales (oms, 2014). En el caso concreto de las mujeres, la hipertensión es un factor de riesgo responsable del 18\% de las muertes en mujeres mayores de 20 años, del 8\% para la diabetes y del 7\% para el sobrepeso (who, 2009).

Los padecimientos crónicos se han asociado con la presencia de sintomatología ansiosa y depresiva (Hervás, Cebolla, \& Soler, 2016), la cual puede contribuir a la presencia de una discapacidad significativa al producirse un efecto de sinergia entre los padecimientos mentales y físicos (Scott et al., 2009; Sharpe \& Curran, 2006; Stein, Cox, Afifi, Belik, \& Sareen, 2006). 
La prevalencia de la sintomatología ansiosa y depresiva se encuentra presente entre un 20 y $35 \%$ en el primer nivel de atención (Bojorquez, Villalobos, Manrique, Tellez, \& Salinas, 2009; Familiar, Borges, Orozco, \& Medina-Mora, 2011) y debido a que se asocia a un nivel de discapacidad, las personas acuden con frecuencia a los servicios médicos (Barlow \& Campbell, 2000; Bojorquez et al., 2009; Mikaliūkštienè et al., 2014).

En México es común que estos síntomas no se identifiquen por diversas razones, entre ellas, la falta de programas enfocados al diagnóstico temprano e identificación de los síntomas o la limitada capacitación en temas de salud mental que recibe el personal de salud en este nivel de atención (Berenzon et al., 2014). Las intervenciones que se han desarrollado se han enfocado en poblaciones clínicas que acuden a niveles especializados de atención con una evolución importante del problema (Riveros, Cortazar-Palapa, Alcazar, \& Sánchez-Sosa, 2005; Sánchez, Velasco, Rodríguez, \& Baranda, 2006; Villamil et al., 2017).

Actualmente el Programa de Reducción de Estrés basado en la atención plena ha demostrado su efectividad en contextos de atención primaria (De Vibe et al., 2015; Goyal et al., 2014; Hervás et al., 2016) para atender diversos padecimientos como los crónico-degenerativos (Bohlmeijer, Prengera, Taala, \& Cuijpersb, 2010; Cherkin et al., 2016; Quintana \& Rincón, 2011); así como para reducir la sintomatología de estrés, ansiedad o depresión (Hoffman, Sawer, Witt, \& Oh, 2010; Strauss, Cavanagh, Oliver, \& Pettman, 2014; Vøllestad, Sivertsen, \& Nielsen, 2011).

El programa se apoya en el supuesto de que factores mentales y emocionales pueden tener un efecto significativo en la salud del individuo (Kabat-Zinn, 2011). Uno de los puntos principales es la reevaluación que las personas hacen acerca de situaciones que las estresan y rebasan, de manera que puedan disminuir el grado de amenaza percibido. Lo anterior se logra a partir de una observación atenta del cuerpo, de las emociones, de los pensamientos y de las conductas.

Uno de los propósitos de la intervención es enseñar a las personas a notar reacciones automáticas y profundamente arraigadas, y cómo éstas, lejos de aportarles un beneficio, sólo alimentan la sensación de amenaza y malestar. La intervención también fomenta un sentido de integración, es decir, lleva a la persona a experimentar directamente la interrelación de sus pensamientos, emociones y conductas. De manera que esta integración se ve reflejada en aspectos de su vida cotidiana. Todo ello mediante el desarrollo de la atención plena (Carmody, 2009).

Entre las características más importantes de la intervención resaltan: 1) Propiciar que sean las personas mismas quienes autogestionen su bienestar a través de contactar sus recursos internos sin depender de nadie y 2) Mostrar que existe una manera accesible para sentirse bien, sin costo y que pueden hacerlo en el momento y lugar que lo requieran (Demarzo et al., 2015).

La mayoría de los resultados de la intervención provienen de estudios realizados en países con poblaciones caucásicas, de un nivel educativo medio y con acceso a servicios de salud (Demarzo et al., 2015; Goyal et al., 2014; Grossman, Nieman, Schmidt, \& Walach 2004; Hoffman et al., 2010). En México, la población que acude al primer nivel de atención tiene características distintas a las mencionadas. Generalmente son mujeres con un nivel socio-económico y educativo bajo que se enfrentan a una serie de barreras para acceder a los servicios de salud mental (Galván, Saavedra, Bartolo, \& Berenzon, 2017).

Surge, entonces, el interés de evaluar si un programa basado en la atención plena es efectivo en una muestra de participantes con características distintas a las que se reportan en la mayoría de los estudios publicados.

Dado lo anterior, el objetivo del presente artículo es evaluar la efectividad de una intervención basada en la atención plena en una muestra de mujeres con enfermedades crónicas y presencia de síntomas de ansiedad y depresión en el primer nivel de atención de la Ciudad de México.

\section{Método}

Se utilizó un diseño cuasi experimental pre-test post-test de un solo grupo (Kerlinger \& Lee, 2002). 
El estudio se realizó en dos fases: 1) La adaptación de la intervención (ver tabla 1) y 2) La evaluación de la efectividad. En este documento únicamente se presentan los datos de la fase 2.

TABLA 1

Fase 1 del estudio correspondiente a la adaptación de la intervención

\begin{tabular}{|c|c|c|}
\hline Objetivo & Método y procedimiento & Resultados \\
\hline \multirow{8}{*}{$\begin{array}{l}\text { Adaptar los materiales, } \\
\text { contenidos, lenguaje } \\
\text { y cuestionario de la } \\
\text { intervención (MAAS) }\end{array}$} & Estudio no experimental, transversal $(n=18)$ & \\
\hline & Se modificó el lenguaje y los contenidos. & \multirow{2}{*}{$\begin{array}{l}\text { La versión final de la intervención fue de } 8 \\
\text { sesiones, se eliminó el tema de la alimentación } \\
\text { y se amplió el tema de las de las relaciones } \\
\text { interpersonales. }\end{array}$} \\
\hline & $\begin{array}{l}\text { Se evaluó la compresión, aceptación } \\
\text { y usabilidad del contenido a través de } \\
\text { cuestionarios autoaplicables. }\end{array}$ & \\
\hline & $\begin{array}{l}\text { Se realizó una validación de los contenidos } \\
\text { de las sesiones a través de expertos en el } \\
\text { tema. }\end{array}$ & \multirow[t]{2}{*}{$\begin{array}{l}\text { Se eliminó el uso de metáforas complejas. Las } \\
\text { sesiones se impartían cada } 2 \text { semanas y su } \\
\text { duración fue de } 1 \text { hora y } 30 \text { minutos. }\end{array}$} \\
\hline & $\begin{array}{l}\text { Se adaptaron y grabaron los audios guía de } \\
\text { las meditaciones. }\end{array}$ & \\
\hline & $\begin{array}{l}\text { Se elaboró un manual del usuario y del } \\
\text { terapeuta, respectivamente, con base en las } \\
\text { modificaciones. }\end{array}$ & \multirow[t]{2}{*}{$\begin{array}{l}\text { Se generó el material necesario: audios, } \\
\text { cuaderno de tareas y una versión final de } \\
\text { ambos manuales. }\end{array}$} \\
\hline & $\begin{array}{l}\text { Se seleccionaron los instrumentos } \\
\text { psicométricos de medición. }\end{array}$ & \\
\hline & $\begin{array}{l}\text { Se tradujo, adaptó y validó } \\
\text { psicométricamente el Mindfulness Attention } \\
\text { and Awareness Scale (MAAS) a la población } \\
\text { de interés. }\end{array}$ & $\begin{array}{l}\text { Se obtuvo la validez y confiabilidad del } \\
\text { MAAS (alfa de Cronbach }=.88 \text {, una varianza } \\
\text { explicada del } 43 \% \text { ) y una estructura factorial } \\
\text { de } 3 \text { factores. La versión final constó de } 13 \\
\text { reactivos. }\end{array}$ \\
\hline
\end{tabular}

\section{Escenario}

Dos centros de salud de primer nivel de atención pertenecientes a la misma alcaldía de la Ciudad de México están localizados en zonas de mediana y alta marginalidad. Atienden principalmente a personas que no cuentan con algún tipo de seguridad social, ambos contaban con las condiciones mínimas necesarias para llevar a cabo la intervención: un espacio físico, mobiliario y equipo.

\section{Participantes}

Una muestra no probabilística por conveniencia de dos centros de salud de la Ciudad de México con una $\mathrm{n}$ inicial de 50 mujeres.

Las mujeres pertenecían a un grupo de pacientes con alguna enfermedad crónica, con el objetivo de monitorear el manejo y tratamiento médico de la enfermedad. Eran mujeres con una media de edad de 59 años. En cuanto a su estado civil, el 70\% de ellas vivía en pareja y el 30\% sin pareja. La mayoría tenía un nivel bajo de escolaridad, 35\% solamente había cursado la primaria; $27 \%$ la secundaria. Con respecto a la ocupación, el $70 \%$ de las mujeres no trabajaba y se dedicaban al hogar, el resto trabajaba como obrera o comerciante. 
En cuanto a la distribución por tipo de padecimiento, el 23\% tenía diabetes mellitus tipo 2, el $23 \%$ tenía hipertensión, el 15\% presentaba obesidad y el 7\% tenía algún otro padecimiento, como problemas óseos.

De las 50 mujeres que iniciaron la intervención, 22 no la concluyeron. Los motivos que mencionaron para no asistir, fueron: a) Falta de tiempo; $b$ ) Tener que atender a algún familiar enfermo o de edad avanzada (madre o suegros);c) Presentar algún problema de salud que les impedía el desplazamiento constante al centro de salud.

\section{Instrumentos}

Los datos fueron recolectados a través de tres escalas al inicio y término de la intervención. A continuación se muestran las escalas:

Perceived Stress Scale (pss) (Cohen, Kamarak, \& Mermelstein, 1983). Adaptada a una muestra mexicana (González \& Landero, 2007). Coeficiente de confiabilidad de 0.83 y una validez convergente con un coeficiente de correlación de 0.553. El punto de corte establecido para el estudio fue $>21$ puntos.

Hospital Anxiety and Depression Scale (hads) (Zigmund \& Snaith, 1983). Se utilizó una versión validada en población mexicana (López-Alvarenga et al., 2002). Son 14 reactivos, 7 para evaluar síntomas ansiosos y 7 para síntomas depresivos. Las respuestas son tipo Likert de 4 opciones. Presenta un alfa de Cronbach de .86 y coeficiente de correlación de .94. el punto de corte establecido para el estudio fue $=0>7$ puntos para síntomas de ansiedad $y=0>6$ para síntomas de depresión.

Mindful Attention and Awareness Scale (maas) (Brown \& Ryan, 2003). Escala de autoreporte de 15 reactivos. Escala tipo Likert de 6 opciones de respuesta. Su validación en población mexicana fue parte de la adaptación de la intervención. Quedó conformada por 13 reactivos, con una confiabilidad de 0.88 y una validez de constructo de $43 \%$ del total de la varianza explicada.

\section{Procedimiento}

Se estableció el contacto con las participantes mediante los trabajadores sociales de los centros de salud. Se realizó una primera sesión introductoria y de evaluación. Se expuso el objetivo, la estructura y la dinámica de la intervención, así como su duración. Se les informó que la intervención formaba parte de una investigación de posgrado de la Facultad de Medicina de la unam. Se les explicó el consentimiento informado, y una vez que lo firmaron, se aplicaron los instrumentos de evaluación de la medición previa a la intervención. $\mathrm{Al}$ inicio de ésta se les entregó a las participantes un folder que contenía un calendario con las fechas de las sesiones, el manual del usuario, un disco con las meditaciones grabadas para practicar en casa y un gafete para identificarlas. Se inició la intervención que, por las condiciones del centro de salud, se impartió quincenalmente de manera grupal, con una duración de hora y media. Las actividades de cada sesión se centraban en el tema correspondiente. Las ocho sesiones tenían en común los ejercicios de meditación, la exposición del tema y la retroalimentación (ver tabla 2). Se asignaron ejercicios para reforzar en casa la práctica de la atención plena. La intervención duró cuatro meses y al finalizar las ocho sesiones, se aplicaron nuevamente los instrumentos de evaluación. 
TABLA 2

Características principales de las ocho sesiones del programa de reducción de estrés basado en la atención plena

Sesión Descripción

\footnotetext{
Bienvenida al programa.

No. 1 Introducción a la práctica meditativa y la atención plena. Introducción a la práctica del escáner corporal y de la respiración.

Las 8 actitudes cultivar en la atención plena.

Exploración de las pautas habituales de pensamiento.

No. 2 Práctica del escáner corporal y de la respiración.

La influencia de las ideas y prejuicios sobre la manera en que las personas se relacionan con su entorno y con ellas mismas.
}

No. 3 Introducción a la práctica de yoga.

El cuerpo como vehículo para estar atento al momento presente.

No. 4 de las personas.

Exploración acerca de qué es el estrés, cómo opera, sus consecuencias y el papel que juega en la reactividad El efecto del estrés a nivel del sistema nervioso.

Práctica del yoga y la respiración.

Exploración de las ventajas de responder al estrés en lugar de reaccionar a él, generando respuestas eficaces en pro del bienestar.

No. 5 Indagación en la manera de reconciliarse con emociones o situaciones desagradables a través de la práctica de la atención plena.

Práctica meditativa en posición sentada.

No. 6

Reconocimiento y amor hacia sí mismos para fomentar la aceptación y afrontamiento eficaz de las emociones y situaciones aflictivas de la vida.

Práctica meditativa en posición sentada.

Práctica meditativa de la bondad como herramienta para trabajar con el miedo.

Énfasis del papel de la atención plena en las relaciones interpersonales para propiciar relaciones más sanas y

No. 7 armoniosas con los demás.

Cualidades para desarrollar la atención plena en la relación con los demás.

Práctica meditativa a elección personal.

Conclusión del programa con énfasis en la práctica de la atención plena en la vida cotidiana.

No. 8 Enfasis en continuar la práctica de la atención plena para incorporarla como una forma de vida.

Plan de acción para continuar con la práctica de la atención plena.

\section{Consideraciones éticas}

El estudio fue aprobado por el Comité de Ética del Instituto Nacional de Psiquiatría Ramón de la Fuente Muñiz (número de comité IRB00006105). Las participantes firmaron el consentimiento informado, el cual incluyó la exposición de riesgos y beneficios de participar en la intervención, el derecho a retirarse de ella sin ninguna consecuencia negativa y la confidencialidad de la información proporcionada. 


\section{Análisis estadístico}

Para el análisis de los datos se utilizó el software spss versión 22. Se empleó estadística exploratoria para conocer la normalidad de los datos con la prueba Shapiro-Wilk, estadística descriptiva, que consistió en las frecuencias, porcentajes, media y desviación estándar, tanto de los datos socio-demográficos como de las variables del estudio. Se realizó una prueba $T$ de Student de grupos relacionados para comparar al grupo antes y después de la intervención. Los resultados se muestran a continuación.

\section{Resultados}

La tabla 3 muestra la comparación de las variables del estudio. A través de una prueba $T$ de Student para dos muestra relacionadas, se evaluó si hubo cambios en las variables de estrés percibido, síntomas ansiosos, síntomas depresivos y la atención plena al finalizar la intervención.

Como se observa en la tabla 3, en las cuatro variables del estudio se presentaron cambios estadísticamente significativos. En la variable de estrés percibido se observa una media de $\chi=26.39$ puntos (antes de la intervención), lo que equivale a una percepción moderada de estrés, misma que se redujo a $\chi=22.75$ (después de la intervención). Este puntaje corresponde cualitativamente haber transitado de un nivel moderado de estrés a un nivel leve.

TABLA 3

Comparación de medias de los puntajes de cada variable antes y después de la intervención

\begin{tabular}{|c|c|c|c|c|c|}
\hline & \multicolumn{2}{|c|}{ Grupo experimental $(n=28)$} & \multirow[t]{3}{*}{$T$} & \multirow[t]{3}{*}{ Gl } & \multirow[t]{3}{*}{$P$} \\
\hline & PRE & POST & & & \\
\hline & $M \quad(D E)$ & $M \quad(D E)$ & & & \\
\hline Estrés percibido & $26.39(6.27)$ & $22.75(7.96)$ & 2.81 & 27 & $.009^{*}$ \\
\hline Síntomas ansiosos & $10.32(3.65)$ & $7.96(3.61)$ & 4.19 & 27 & $.000^{\star *}$ \\
\hline Sintomas depresivos & $7.57(3.73)$ & $6.28(3.63)$ & 2.73 & 27 & $.011^{*}$ \\
\hline Atención plena & $46.50(14.99)$ & $53.85(14.62)$ & -3.23 & 27 & $.003^{*}$ \\
\hline
\end{tabular}

${ }^{*} \mathrm{P}<.05^{* *} \mathrm{P}<.001$

Para las variables de síntomas ansiosos y depresivos, también se observa una disminución en el puntaje promedio de ambas. En el caso de los síntomas de ansiedad, el puntaje medio disminuyó de $\chi=10.32$ al inicio de la intervención, a uno de $\chi=7.96$ al finalizarla, mostrando una diferencia estadísticamente significativa menor a $\alpha .001$. Para los síntomas de depresión la disminución fue baja (de $\chi=7.57$ a $\chi=6.28)$. Por otro lado, el puntaje promedio de atención plena aumentó de $\chi=46.50$ al inicio a $\chi=53.85$ al finalizar la intervención.

Finalmente se calculó el tamaño del efecto (te) para evaluar el tamaño de los cambios presentados. En la variable de estrés percibido, el te presentó una d de Cohen=.46 $(\mathrm{r}=.25)$, para los síntomas de ansiedad se obtuvo una d de Cohen=.65 ( $\mathrm{r}=.31$ ) y para los síntomas de depresión, una d de Cohen=.35 ( $\mathrm{r}=.17)$. De acuerdo con Goyal et al., (2014), dichos tamaños del efecto se consideran de bajos a moderados y son similares a los observados en tratamiento farmacológico.

Como lo demuestran los resultados, todas las variables dependientes resultaron con cambios estadísticamente significativos antes y después de la intervención. Los síntomas de depresión fueron los que presentaron puntajes promedio más bajos tanto al inicio como al final de la intervención. Por otro lado, la variable que 
muestra un nivel de significancia mayor es la sintomatología ansiosa. Del mismo modo, se corrobora un aumento en la capacidad de atención plena.

\section{Discusión}

Las investigaciones realizadas en primer nivel son escasas comparadas con niveles especializados (Dermazo et al., 2015) por lo que los resultados de este estudio constituyen uno de los intentos en México por conocer los alcances de este tipo de intervenciones para tratar la salud mental. El estudio se enfocó en enfermedades crónicas como diabetes e hipertensión, mismas que son altamente prevalentes entre la población mexicana femenina (Rojas et al., 2018).

Uno de los aspectos más importantes a destacar en este estudio es, además del escenario, el tipo de población con la que se trabajó. Un grupo de mujeres con una enfermedad crónica, baja escolaridad y bajos recursos económicos. Condiciones que son muy disimiles a las características sociodemográficas promedio de las muestras reportadas en otros estudios (Demarzo et al., 2015; Goyal et al., 2014; Grossman et al., 2004; Hoffman et al., 2010; Toneatto \& Nguyen, 2007). Donde generalmente se interviene a una población con un nivel educativo medio, con recursos y acceso a atención en salud y con mayores ingresos. Lo que hace notar que aún bajo condiciones adversas, estas mujeres aprendieron estrategias de autoregulación para el manejo de su estrés y síntomas relacionados, beneficiándose del trabajo realizado en la intervención, al igual que lo reportado en distintos países con una población mayoritariamente de mujeres (Dermazo et al. 2015). Esto es particularmente relevante si se consideran las barreras para la atención en salud mental que existen actualmente en México (Galván et al., 2017).

Las participantes que concluyeron la intervención mencionaron haber sentido una mejoría visible en su estado de ánimo, se sentían motivadas a asistir, les agradaba y les ayudaba los temas abordados en las sesiones de manera que podía manejar de mejor manera algún problema cotidiano.

De acuerdo con Goyal et al., (2014), tanto el estrés como la sintomatología ansiosa y depresiva se consideran distintos componentes del afecto negativo. Los autores mencionan que, a través una intervención basada en la atención plena, se propicia una mejoría de dicho afecto negativo. Así lo demuestran los resultados obtenidos en este estudio: una disminución moderada de los síntomas de estrés, ansiedad y depresión como uno de los principales efectos de la intervención, evidencia que es similar a los encontrado por otros estudios (Hoffman et al., 2010; Strauss et al., 2014; Vøllestad et al., 2011).

Se observó un aumento en la atención plena, tal y como se ha reportado en otros estudios (Bohlmeijer et al., 2010; Brown, Ryan, \& Creswell, 2007; Quintan \& Rincón, 2011). El aumento en la capacidad de estar atentos, tanto de manera focal como integral, es uno de los objetivos principales de intervenciones de este tipo (Bishop et al., 2004; Carmody, 2009), pues es a partir de la observación atenta que se logra una repercepción de la experiencia.

El tamaño del efecto encontrado en este estudio para el estrés percibido y la sintomatología ansiosa fue moderado, para la sintomatología depresiva fue bajo, lo cual es similar a lo reportado en otros estudios (Bohlmei-jer et al., 2010; Demarzo et al., 2015; Goyal et al., 2014).

Esto puede atribuirse a que, dado que es un primer estudio, aún requiere afianzarse elementos metodológicos a fin de asegurar un mayor tamaño del efecto y también a que la investigación de la intervención en el primer nivel de atención aún se encuentra en desarrollo (Demarzo et al., 2015).

Esta intervención representa un recurso útil para una población mexicana donde los servicios de salud mental son escasos (Berenzon et al., 2014; Galván et al., 2017) y los que existen se encuentran saturados o son poco adecuados para la población (Goyal et al., 2014). Es una intervención autodirigida, de bajo costo y de fácil acceso que le devuelve el poder a las personas de ser ellas mismas quienes gestionen su bienestar. 


\section{Conclusión}

Una intervención basada en la atención plena en el primer nivel de atención resultó efectiva en un grupo de mujeres con alguna enfermedad crónica y presencia de sintomatología ansiosa y depresiva. Ello, a pesar de las restricciones educativas y económicas de las participantes, así como de las limitaciones del acceso a servicios de salud mental.

\section{Limitaciones y sugerencias}

Debido a que los datos aquí presentados pertenecen a un primer estudio, existen algunos elementos a considerar como parte de las limitaciones de los resultados. El primero de ellos tiene que ver con el sesgo muestral o de selección. La manera en que se asignaron las participantes a la intervención fue intencional y no aleatoria debido a las condiciones del contexto, por lo que se recomienda realizar una asignación aleatoria para otros estudios. La segunda limitación se relaciona con la falta de un grupo control equivalente a manera de establecer comparaciones en el efecto de la intervención entre los grupos. La inclusión de un dicho grupo sería recomendable en futuros estudios. Finalmente se requiere de una evaluación que considere mayor rigor metodológico que permita resultados más precisos y robustos.

\section{Agradecimientos}

Las autoras expresamos nuestro reconocimiento a la Secretaría de Salud del Distrito Federal y en especial a las personas entrevistadas que compartieron sus experiencias para hacer posible este trabajo.

\section{REFERENCIAS}

Barlow, D., \& Campbell, L. (2000). Mixed anxiety-depression and its implications for models of mood and anxiety disorders. Comprehensive Psychiatry, 41(2), 55-60. doi:10.1016/S0010-440X(00)80009-7

Berenzon, S. B., Galván, J., Saavedra, N., Bernal, P., Mellor-Crummey, L., \& Tiburcio, M. (2014). Exploración del malestar emocional expresado por mujeres que acuden a centros de atención primaria de la Ciudad de México. Un estudio cualitativo. Salud Mental, 37(4), 313-319.

Bishop, S., Lau, M., Shapiro, S., Carlson, L., Anderson, N., Carmody, J., \& Devins, G. (2004). Mindfulness: A proposed operational definition. Clinical Psychology: Science and Practice, 11(3), 230-241. doi:10.1093/ clipsy.bph077

Bohlmeijer, E., Prengera, R., Taala, E., \& Cuijpersb, P. (2010). The effects of mindfulness-based stress reduction therapy on mental health of adults with a chronic medical disease: A meta-analysis. Journal of Psychosomatic Research, 68, 539-544. doi:10.1016/j. jpsychores.2009.10.005

Bojorquez, I., Villalobos, V., Manrique, B., Tellez, M., \& Salinas, A. (2009). Depressive symptoms among poor older adults in Mexico: Prevalence and associated factors. Panamerican American Journal of Public Health, 26(1), 70-76.

Brown, K., \& Ryan, R. (2003). The benefits of being present: Mindfulness and its role in psychological well-being. Journal of Personality and Social Psychology, 84(4), 822-48. doi:10.1037/0022-3514.84.4.822

Brown, K., Ryan, R., \& Creswell, J. (2007). Mindfulness: Theoretical foundations and evidence for its salutary effects. Psychological Inquiry, 18, 211-237. doi:10.1080/10478400701598298

Carmody, J. (2009). Evolving conceptions of mindfulness in clinical settings. Journal of Cognitive Psychotherapy: An International Quarterly, 23(3), 270-280. doi:10.1891/0889-8391.23.3.270

Cherkin, D., Sherman, K., Balderson, B., Cook, A., Anderson, M., Hawkes, R., \& Turner, J. (2016). Effect of mindfulness-based stress reduction vs cognitive behavioral therapy or usual care on back pain and functional 
limitations in adults with chronic low back pain: A randomized clinical trial. jama, 315, 1240- 1249. doi:10.1001/jama.2016.2323

Cohen, S., Kamarak, T., \& Mermelstein, R. (1983). A global measure of perceived stress. Journal of Health and Social Behavior, 24(4), 385-396. doi:10.2307/2136404

De Vibe, M., Solhaug, I., Tyssen, R., Friborg, O., Rosenvinge, J. H., Sørlie, T., \& Bjørndal, A. (2015). Does personality moderate the effects of mindfulness training for medical and psychology students? Mindfulness, 6(2), 281-289. doi:10.1007/s12671-013-0258-y

Demarzo, M., Montero-Marin, J., Cuijpers, P., Zabaleta, E., Mahtani, K, Velling, A., Vicens, C., López-del- Hoyo, Y., \& García-Campayo, J. (2015). The efficacy of mindfulness-based interventions in primary care: A meta-analytic review. Annals of Family Medicine 13(6), 573-583. doi:10.1370/afm.1863

Familiar, I., Borges, G., Orozco, R., \& Medina-Mora, M. E. (2011). Mexican migration experiences to the US and risk for anxiety and depressive symptoms. Journal of Affective Disorders, 130(1-2), 83-91. doi:10.1016/j. jad.2010.09.025

Galván, J., Saavedra, N., Bartolo, F., \& Berenzon, Sh., (2017). Perceptions of Mexican women regarding barriers in mental Health Services in primary care. bmc Women's Health, 17(70), 1-6. doi:10.1186/ s12905-017-0423-x

González, M., \& Landero, R. (2007). Factor structure of the perceived stress scale (pss) in a sample from Mexico. The Spanish Journal of Psychology, 10(1), 199-206. doi:10.1017/S1138741600006466

Goyal, M., Singh, S., Sibinga, E., Gould, N., Rowland- Seymour, A., Sharma, R., \& Ranasinghe, P. (2014). Meditation programs for psychological stress and well-being: A systematic review and meta-analysis.jama Internal Medicine, 174(3), 357-368. doi:10.1001/ jamainternmed.2013.13018

Grossman, P., Nieman, L., Schmidt, S., \& Walach, H. (2004). Mindfulness-based stress reduction and health benefits. A meta-analysis. Journal of Psychosomatic Research, 57(1), 35-43. doi:10.1016/S0022- 3999(03)00573-7

Hervás, G., Cebolla, A., y Soler, J. (2016). Intervenciones psicológicas basadas en mindfulness y sus beneficios: Estado actual de la cuestión. Clínica y Salud, 27(3), 115-124. doi:10.1016/j.clysa.2016.09.002

Hoffman, S., Sawer, A., Witt, A., \& Oh, D. (2010). The effect of mindfulness-based therapy on anxiety and depression: A meta-analytic review. Journal of Consulting and Clinical Psychology, 78(2), 169-183. doi:10.1037/a0018555

Kabat-Zinn, J. (2011). Vivir con plenitud las crisis. Barcelona, España: Kairós.

Kerlinger, F., \& Lee, H. (2002). Investigación del comportamiento. Métodos de investigación en ciencias sociales. México: Mc Graw Hill.

López-Alveranga, J., Vázquéz-Velázquez, V., Arcila-Martínez, D., Sierra-Ovando, A., González- Barranco, J., \& SalinPascual, R. (2002). Exactitud y utilidad diagnóstica del Hospital Anxiety and Depression Scale (hads) en una muestra de sujetos obesos mexicanos. Revista de Investigación Clínica, 54(5), 403-409.

Mikaliūkštienė, A., Žagminas, K., Juozulynas, A., Narkauskaitė, L., Sąlyga, J., Jankauskienė, K., Stukas, R., \& Šurkienė, G. (2014). Prevalence and determinants of anxiety and depression symptoms in patients with type 2 diabetes in Lithuania. Medical Science Monitor, 20, 182-190. doi:10.12659/MSM.890019

Organización Mundial de la Salud [oms] (2014). Informe sobre la situación mundial de las enfermedades no transmisibles 2014. Recuperado de https://www.who.int/nmh/publications/ncd-status-report-2014/es/

Quintana, M., \& Rincón, M. (2011). Eficacia del entrenamiento en mindfulness para pacientes con fibromialgia. Clínica y Salud, 22(1), 51-67.

Riveros, A., Cortazar-Palapa, J., Alcazar, F., \& Sánchez-Sosa, J. (2005). Efectos de una intervención cognitivoconductual en la calidad de vida, ansiedad, depresión y condición médica de pacientes diabéticos e hipertensos esenciales. International Journal of Clinical and Health Psychology, 5(3), 445-462.

Rojas, R., Basto, A., Aguilar, C, Zarate, E., Villalpando, S., \& Barrientos, T. (2018). Prevalencia de diabetes por diagnóstico médico previo en México. Salud Pública de México, 60(3), 224-232.

Sánchez, S., Velasco, L., Rodríguez, R., \& Baranda, J. (2006). Eficacia cognitivo-conductual para la disminución de la ansiedad en pacientes enfermos. Revista de Investigación Clínica, 58(6), 540-546. 
Scott, K., Von Korff, M., Alonso, J., Angermeyer, M., Bromet, E., Fayyad, J., de Girolamo, G., Demyttenaere, K., Gasquet, O., Gureje., Haro, J. M., He, Y., Kessler, R. C., Levunson, D., Medina Mora, M. E., Oakley Browne, M., Ormel, J., Posada-Villa, J., Watanabe, M., \& Williams, D. (2009). Mental-physical co-morbidity and its relationship with disability: Results from the World Mental Health Surveys. Psychological Medicine, 39(1), 33-43. doi:10.1017/S0033291708003188

Sharpe, L., \& Curran, L. (2006). Understanding the process of adjustment to illness. Social Science and Medicine, 62(5), 1153-1166. doi:10.1016/j.socscimed.2005.07.010

Stein, M., Cox, B., Afifi, T., Belik, S., \& Sareen, J. (2006). Does co-morbid depressive illness magnify the impact of chronic physical illness: A population-based perspective. Psychological Medicine, 36, 587-596.

Strauss, C., Cavanagh, K., Oliver, A., \& Pettman, D. (2014). Mindfulness-based interventions for people diagnosed with a current episode of an anxiety or depressive disorder: A meta-analysis of randomized controlled trials. PLoS One, 9(4), e96110. doi:10.1371/ journal.pone.0096110

Toneatto, T., \& Nguyen, L. (2007). Does mindfulness meditation improve anxiety and mood symptoms? A review of the controlled research. The Canadian Journal of Psychiatry, 52(4), 260-266. doi:10.1177/070674370705200409

Villamil, V., Vargas, B., Caraveo, J., González, J., Díaz, A., Cortés., \& Pérez, M. (2017). Glucose and cholesterol stabilization in patients with type 2 diabetes mellitus with depressive and anxiety symptoms by problem-solving therapy in primary care centers in Mexico City. Primary Health Care Research and Development, 19(1), 1-9. doi: $10.1017 /$ S1463423617000512

Vøllestad, J., Sivertsen, B., \& Nielsen, G. (2011). Mindfulness-based stress reduction for patients with anxiety disorders: Evaluation in a randomized controlled trial. Behaviour Research and Therapy, 49(4), 281-288. doi:10.1016/j.brat.2011.01.007

World Health Organization [who] (2009). Women and health. Today's evidence tomorrow's agenda (who Report). Geneva, Switzerland: who.

Zigmund, A., \& Snaith, R. (1983). The hospital anxiety and depression scale. Acta Psiquiátrica Scandinavica, 67(6), 361-70. doi:10.1111/j.1600-0447.1983. tb09716.x

\section{Notas}

1 La Ciudad de México se divide, para su gestión política y geográfica, en lo que se denomina 'alcaldías'. Son un total de 16 y cada una cuenta con sus propios servicios en salud. 